\title{
Ictiofauna de los humedales del río San Pedro, Balancán, Tabasco, México
}

\author{
Alfonso Castillo-Domínguez ${ }^{1 *}$, Everardo Barba Macías ${ }^{1}$, Alberto de Jesús Navarrete ${ }^{2}$, \\ Rocío Rodiles-Hernández ${ }^{3}$ \& María de Lourdes Jiménez Badillo ${ }^{4}$ \\ 1. El Colegio de la Frontera Sur, Unidad Villahermosa, Depto. de Aprovechamiento y Manejo de Recursos Acuáticos. \\ Carretera Villahermosa-Reforma km. 15.5, Ranchería Guineo 2a sección C.P. 86280 Villahermosa, Tabasco, México; \\ alfonsin75@gmail.com, ebarba@ecosur.mx \\ 2. El Colegio de la Frontera Sur, Unidad Chetumal. A.P. 424,77000 Chetumal, Quintana Roo, México; \\ anavarrete@ecosur.mx \\ 3. El Colegio de la Frontera Sur, Unidad San Cristóbal. A.P. 63 San Cristóbal de Las Casas, Chiapas, México; \\ rrodiles@ecosur.mx \\ 4. Instituto de Ciencias Marinas y Pesquerías. Universidad Veracruzana. Hidalgo 617. Col. Río Jamapa. C.P. 94290 Boca \\ del Río, Veracruz, México; ljimenez@uv.mx \\ * Autor de correspondencia.
}

Recibido 29-VI-2010. Corregido 20-X-2010. Aceptado 23-XI-2010.

\begin{abstract}
Ichthyofauna from wetlands of San Pedro, Balancán, Tabasco, México. San Pedro River's wetlands sustain trophic nets in the fluvial system, due to the high habitat availability, and space and temporal variations. In order to describe the relationship between environmental parameters and ichthyofauna, this study evaluated fish assemblages composition, distribution, abundance, density, biomass, richness species, diversity and equitability in the wetlands. Sampling considered three different sites and climatic seasons (dry, rainy and cold fronts). The physical and chemical parameters considered were dissolved oxygen, temperature, $\mathrm{pH}$, water transparency and the depth. Fishes were caught with a shrimp net, after six minutes towings and were identified afterwards. A total of 1049 organisms of 25 fish species were caught, two of which were exotic species: Oreochromis niloticus and Parachromis managuense. A total of 23 species were found at site I (with the highest density 0.23 ind.$\left./ \mathrm{m}^{2}\right), 17$ at site II $\left(0.23\right.$ ind $\left./ \mathrm{m}^{2}\right)$ and 14 at site III $\left(0.12 \mathrm{ind} . / \mathrm{m}^{2}\right)$. The dry season had the highest species number with 21 species, followed by the rainy season with 17 species, and the cold season with five species. Similarly, the highest biomass $\left(8.30 \mathrm{~g} / \mathrm{m}^{2}\right)$ was found in dry season, followed by the rainy $\left(2.16 \mathrm{~g} / \mathrm{m}^{2}\right)$ and the cold seasons $\left(0.03 \mathrm{~g} / \mathrm{m}^{2}\right)$. Considering seasons, highest density was found during the dry $\left(0.436 \mathrm{ind} . / \mathrm{m}^{2}\right)$, followed by the rainy $\left(0.213\right.$ ind.$\left./ \mathrm{m}^{2}\right)$ and the cold $\left(0.023 \mathrm{ind} . / \mathrm{m}^{2}\right)$ seasons. The dominant density species during the study period, according to the quadrants graphic of Olmstead-Tukey were: Petenia splendida, Vieja heterospila, Vieja synspila, Dorosoma petenense and Astyanax aeneus. There were significant differences in the species richness among sites. Temperature, depth and transparency showed differences among the seasons. The canonical correspondence analysis indicated that fish distribution was governed by environmental parameters during all seasons. In terms of fish abundance and composition, environmental parameters play an important role showing spatial and temporal differences in the ecosystem, this could be explained with the fact that most of young fishes have a movement behavior to the wetlands, searching refuge and feed during the dry season. Considering the diversity indexes variation, it may be concluded that San Pedro River's wetlands correspond to a system where the ichthyofauna composition fluctuates spatial and seasonally. Rev. Biol. Trop. 59 (2): 693-708. Epub 2011 June 01.
\end{abstract}

Key words: biomass, density, dominance, richness specific, wetlands of the San Pedro River. 
Los humedales están definidos como sistemas intermedios o de transición entre los ecosistemas acuáticos y terrestres, donde la masa de agua es somera (Cowardin et al. 1979). La Convención Ramsar los define como "Extensiones de marismas, pantanos y turberas, o superficies cubiertas de aguas, sean éstas de régimen natural o artificial, permanentes o temporales, estancadas o corrientes, dulces, salobres o saladas, incluidas las extensiones de agua marina cuya profundidad en marea baja no exceda de seis metros" (Anónimo 1999).

Las funciones ecológicas de los humedales se derivan de sus características hidrológicas, geológicas y biológicas. Entre las funciones y valores reconocidos se incluyen el constituir un hábitat para la vida acuática y terrestre, lugares de enseñanza e investigación, reciclaje y transformación de nutrientes, alteración de los flujos de inundación, recarga de acuíferos, retención de partículas, elevada productividad, recreación y estabilización del suelo (Kent 2000).

Estas funciones explican la alta disponibilidad del hábitat y nivel de relaciones tróficas que sostienen por los suministros de carbono orgánico, debido a las variaciones espaciales y temporales del ambiente (Junk \& Welcomme 1990, Mistch \& Gosselink 2000), así como a aquellos parámetros asociados a la ontogenia, reproducción y/o alimentación de muchas especies de peces dulceacuícolas (Welcomme 1985, Methven et al. 2001, Taylor \& Warren 2001). Pese a la importancia de los humedales en Tabasco, la mayoría de los esfuerzos científicos y de investigación se han enfocado a la Reserva de la Biosfera Pantanos de Centla, donde se han realizado inventarios, listados taxonómicos y evaluado el uso de diferentes hábitats en la zona del delta Grijalva-Usumacinta (Páramo 1984, Reséndez \& Salvadores 2000, Macossay 2008). Por otro lado, la cuenca del Usumacinta es un área hidrológica y ecológicamente importante, debido a la presencia de zonas aún conservadas, sin embargo, son pocos los trabajos científicos realizados, en los cuales se destaca el estudio ecológico de los peces del río San Pedro y su zona de inundación, así como los ciclos de vida de algunas especies de peces con interés para la piscicultura (Chávez et al. 1988). Asimismo, Espinosa-Pérez \& DazaZepeda (2005), presentaron un listado de 95 especies, de las cuales nueve son endémicas o exclusivas de las cuencas en el estado de Tabasco y recientemente, el monitoreo de peces e invertebrados en ambientes lóticos y lénticos de Balancán (Estrada 2008).

El presente trabajo, contribuye al conocimiento de la composición taxonómica y ecológica de los peces de aguas interiores, mediante la determinación de la densidad, biomasa, diversidad, riqueza específica y equidad, así como su relación espacio-temporal con las variables ambientales en la zona de inundación del río San Pedro.

\section{MATERIALES Y MÉTODOS}

Área de estudio: Los humedales del río San Pedro pertenecen a la región Usumacinta. Este río nace en las montañas del Petén en Guatemala y se adentra a México por la parte sur de Tabasco, donde se dirige hacia el norte y guarda esta dirección hacia el poblado de San Pedro, para continuar hacia el oeste y desembocar en el río Usumacinta. Entonces, se localiza en los municipios de Balancán y Tenosique, colinda al sur y al este con la República de Guatemala, al norte con el estado de Campeche, al este con el municipio de Emiliano Zapata y el estado de Chiapas. Por lo tanto, tiene un escurrimiento aproximado de $50719 \mathrm{x}$ $10^{6} \mathrm{~m}^{3}$ al año (INEGI 2006). La subcuenca de San Pedro está compuesta por rocas calcáreas del Cretácico y del Terciario que provocan una coloración verde claro en el agua y una débil carga de materia en suspensión (0.2 millones de $\mathrm{m}^{3}$ por año) (West et al. 1985). El grado de inundación de los humedales está condicionado por las épocas climáticas. La época de lluvias establecida de mayo a octubre, con temperatura media de $32^{\circ} \mathrm{C}$ y una precipitación pluvial de $338 \mathrm{~mm}$. La época de nortes que va de noviembre a enero, con una temperatura media de $20^{\circ} \mathrm{C}$ y finalmente, la época de secas de febrero a abril con temperatura y precipitación media 
de $36^{\circ} \mathrm{C}$ y $6.8 \mathrm{~mm}$, respectivamente (INEGI 2006, Moguel \& Molina-Enríquez 2000).

Los tipos de vegetación dominante en la zona son: selva alta y baja perennifolia, pastizales de cultivo, sabana, palmar inundable y una alta variedad de hábitats acuáticos como: pantanos, ríos y lagunas (Anónimo 2002). La vegetación circundante se encuentra constituida por vegetación hidrófila emergente, Cyperus articulatus, Thypa latifolia, Thalia geniculata, Cladium jamaicense; emergente enraizada, Acelorrhaphe wrightii (tasiste); selva baja inundable, Pachyra aquatica (apompo), Haematoxyllum campechianum (tinto) (Barba et al. 2006).
Trabajo de campo: El área de estudio esta en la zona de inundación del río San Pedro en Balancán. Los muestreos se realizaron durante horas de iluminación en tres sitios (sitio I: $91^{\circ} 08^{\prime} 02^{\prime \prime} \mathrm{N}-17^{\circ} 46$ ' $59^{\prime}$ " W; sitio II: $91^{\circ} 07^{\prime} 35^{\prime}$ " $\mathrm{N}-17^{\circ} 46^{\prime} 09^{\prime \prime} \mathrm{W}$; sitio III: $91^{\circ} 08^{\prime} 18^{\prime \prime} \mathrm{N}$ $17^{\circ} 43^{\prime} 16^{\prime}$ 'W) (Fig. 1), que corresponden a drenes artificiales, los cuales atraviesan la zona de inundación construidos por el gobierno en los años setentas con fines de riego para agricultura y ganadería (Plan Balancán-Tenosique) (Fig. 1). La recolección de peces se efectúo en tres épocas climáticas: lluvias (junio-septiembre, 2007), nortes (noviembre-enero, 2007-2008) y secas (febrero-abril, 2008). En cada época

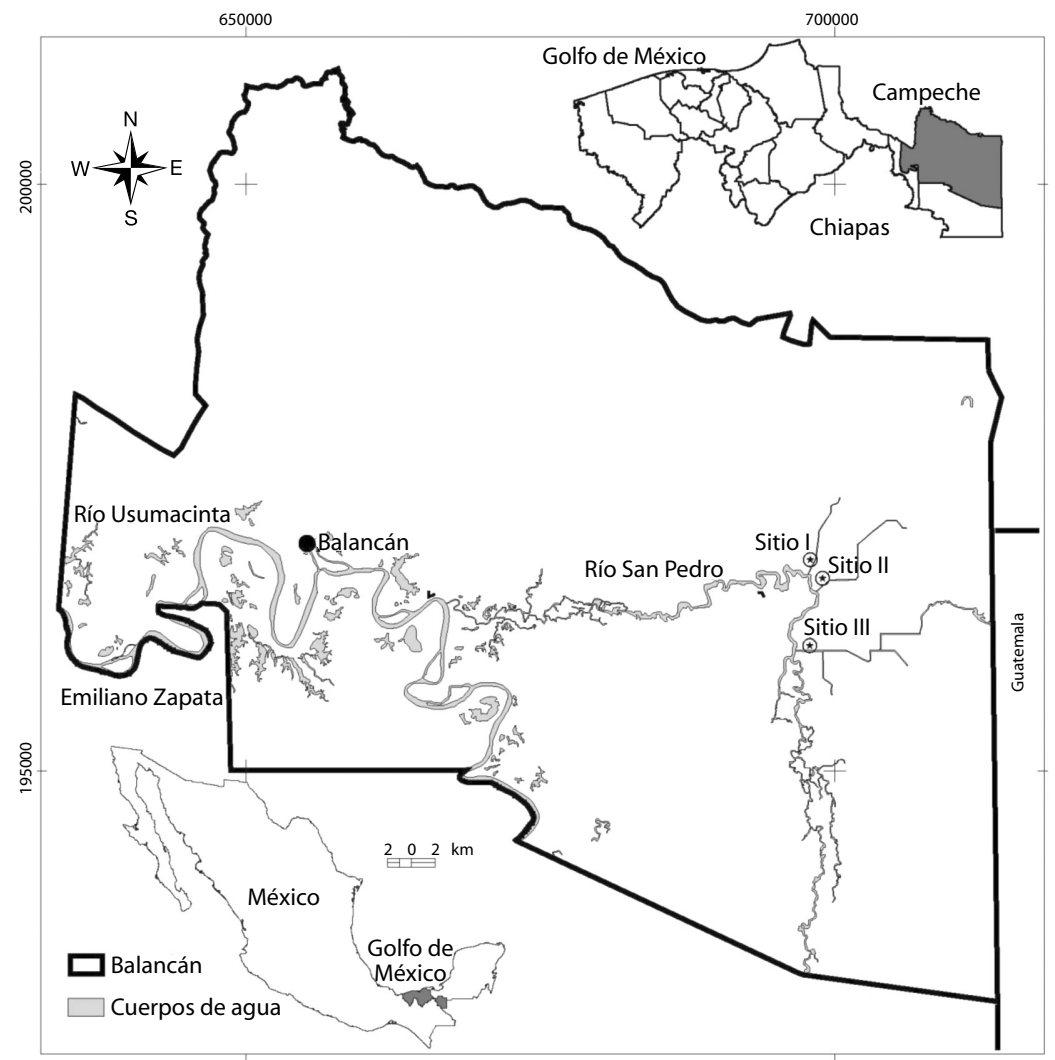

Fig. 1. Área de estudio y sitios muestreados.

Fig. 1. Study area and sampled sites. 
climática se realizaron tres salidas mensuales con una duración de cinco días para la recolección de organismos.

En cada sitio se determinó el oxígeno disuelto $(\mathrm{mg} / \mathrm{l})$, la temperatura $\left({ }^{\circ} \mathrm{C}\right)$ y el $\mathrm{pH}$ a una profundidad media, en donde se utilizó un multianalizador HANNA HI-9828; la profundidad $(\mathrm{cm})$ se registró con una cuerda graduada y la transparencia de la columna de agua $(\mathrm{cm})$ mediante un disco de Secchi. Las muestras biológicas se obtuvieron con una red de prueba camaronera de $7.25 \mathrm{~m}$ de largo, $5.20 \mathrm{~m}$ de abertura de boca con una luz de malla $1.9 \mathrm{~cm}$. La duración de cada arrastre fue de 6 minutos en cada sitio de muestreo. El área de barrido por el arte de pesca se calculó con la obtención del producto de la velocidad del arrastre por el tiempo de operación y por la abertura del trabajo de la red (Stevenson 1982), y cubrió un área de arrastre de $1404 \mathrm{~m}^{2}$. Las muestras obtenidas se fijaron en formalina al $10 \%$ y posteriormente, en el laboratorio se lavaron con agua y fueron conservadas en alcohol etílico al 70\%. Para la identificación taxonómica se utilizaron las claves convencionales de peces dulceacuícolas (Álvarez 1970, Schmitter-Soto1998, Miller et al. 2005).

La estructura de la comunidad se analizó mediante la densidad relativa, la cual fue cuantificada como el número de organismos muestreados dividido entre el área de barrido; mientras que la biomasa fue expresada como el peso húmedo fijado en gramos (phf) entre el área de barrido, para ello todos los organismos fueron pesados individualmente con una balanza digital $( \pm 0.001 \mathrm{~g})$. Para la jerarquización de las especies por su densidad (ind. $/ \mathrm{m}^{2}$ ), biomasa $\left(\mathrm{g} / \mathrm{m}^{2}\right)$ y frecuencia (expresada \%), se aplicó la prueba de asociación no paramétrica de tipo Olmstead-Tukey denominada gráfico de cuadrantes (Sokal \& Rohlf 1981), mediante éste análisis se obtuvieron las siguientes categorías: 1) Dominantes: Densidad y frecuencia alta (especies que presentaron valores de densidad, biomasa y frecuencia mayores a la media, 2) Constantes: Densidad baja y frecuencia alta (especies cuyo valor de densidad, biomasa es menor a la media y su frecuencia es mayor a la media), 3) Frecuentes: Densidad alta y frecuencia baja (especies cuyo valor de densidad, biomasa es mayor y su frecuencia es menor a la media), y 4) Ocasionales o raras: Densidad y frecuencia baja (especies cuyo valor de densidad, biomasa y frecuencia es menor a la media). Los parámetros ecológicos de la comunidad se analizaron a nivel espacial y por épocas en el cual se consideraron los principales descriptores: diversidad en bits ind. $/ \mathrm{m}^{2}\left(\mathrm{H}^{\prime}\right)$ (Shannon \& Wiener 1963), riqueza de especies (S) (Margalef 1969), equidad (J’) (Pielou 1966).

Los parámetros físico-químicos así como los parámetros ecológicos de la comunidad se compararon entre los sitios (I, II y III) y entre épocas climáticas (secas, lluvias y nortes). La comparación de los promedios entre las muestras fue corroborada bajo el supuesto de la homogeneidad de varianza mediante la prueba de Levene (1960). Los datos con varianza homogénea entre grupos se evaluaron mediante el análisis de varianza (ANDEVA) de una vía $(F)$ con un nivel de significancia de $(\mathrm{p}<0.05)$ (Daniel 2008); cuando se presentaron diferencias significativas se procedió a determinar entre que sitio y época se mostró la diferencia en el parámetro ambiental o ecológico y se utilizó la prueba de comparaciones múltiples de Tukey, la cuál es más robusta para muestras que no presentan el mismo número de datos (Dunnett 1980, Zar 1999). Los análisis estadísticos se realizaron con el paquete STATISTICA versión 7.0 para Windows (StatSoft 2004). Asimismo, se realizó un análisis de correspondencia canónica (ACC) para determinar las relaciones entre las especies y las variables ambientales. Este método se emplea para extraer gradientes ambientales sintéticos de grupos de datos ecológicos. Estos gradientes son la base para describir y visualizar las preferencias de las especies por diferentes hábitats representados en un diagrama de ordenación que asumen la distribución de las especies como una función Gaussiana de las variables ambientales (Ter Braak \& Verdonshot 1995). Este método fue elegido debido a que es apropiado cuando el objetivo es describir cómo 
responden las especies a un conjunto de variables ambientales observadas. El análisis fue realizado con el programa Multivariate Statistical Package versión 3.12 (MVSP 1985-2000).

\section{RESULTADOS}

Parámetros ambientales: Las variaciones espacio-temporales de los parámetros físicoquímicos de la zona de inundación del río San Pedro se resume en el Cuadro 1. No existieron diferencias significativas en las variables ambientales para los sitios I, II y III ( $\mathrm{F}=2.61$, $\mathrm{p}>0.05$ ); sin embargo, los sitios que presentaron promedios mayores de concentración de oxígeno disuelto, temperatura y $\mathrm{pH}$ fueron los sitios I y II. Se observaron cambios en los niveles del agua, y se obtuvieron estos cambios en los sitios I y II, con profundidades de $166 \mathrm{~cm}$ y $165 \mathrm{~cm}$, mientras la transparencia se encuentra en función de las condiciones del día, y está relacionada con la productividad primaria, esta variable presentó los promedios mínimos en los sitios I y III. Con respecto a la variabilidad ambiental durante las épocas evaluadas, el oxígeno disuelto y la temperatura mostraron diferencias significativas $(\mathrm{F}=370.7$, $\mathrm{p}<0.05 ; \mathrm{F}=6.206, \mathrm{p}<0.05)$, tales diferencias se observaron en las épocas de lluvias y nortes (Tukey, $\mathrm{p}<0.05$ ). Los valores promedios del $\mathrm{pH}$ por épocas no presentaron diferencias significativas, el promedio mínimo se presentó en la época de lluvias (7.2), y el promedio máximo en nortes (7.7), y se mantuvieron estos valores ligeramente alcalinos. Por otro lado, existieron diferencias significativas $(\mathrm{F}=8.87, \mathrm{p}<0.05)$ en los promedios de la profundidad entre las épocas de secas $(131 \mathrm{~cm})$ y nortes $(186 \mathrm{~cm})$ (Tukey, $\mathrm{p}<0.05$ ).

Composición ictiofaunística: En los sitios I, II y III de los humedales del río San Pedro, la riqueza estuvo representada por peces jóvenes y adultos. Entonces, se capturaron un total de 1049 individuos, pertenecientes a nueve familias, 19 géneros y 25 especies (Cuadro 2). En el sitio I ( $\mathrm{n}=178)$, se identificaron 23 especies correspondientes a ocho familias y 16 géneros;
CUADRO 1

Parámetros ambientales de los sitios I, II y III de los humedales en San Pedro, Balancán, Tabasco, México

TABLE 1

Environmental parameters of the sites I, II, III at wetlands San Pedro, Balancán, México

\begin{tabular}{|c|c|c|c|c|}
\hline \multirow{3}{*}{ Épocas } & \multicolumn{3}{|c|}{ Sitios } & \multirow{2}{*}{ Promedio } \\
\hline & I & II & III & \\
\hline & \multicolumn{4}{|c|}{ Oxígeno disuelto (mg/l) } \\
\hline Lluvias & $* * * * *$ & $* * * * *$ & $* * * * *$ & $* * * * *$ \\
\hline Nortes & 3.9 & 3.7 & 3.9 & 3.8 \\
\hline Secas & 6.7 & 6.8 & 5.9 & 6.5 \\
\hline \multirow[t]{2}{*}{ Promedio } & 3.5 & 3.4 & 3.2 & \\
\hline & \multicolumn{4}{|c|}{ Temperatura $\left({ }^{\circ} \mathrm{C}\right)$} \\
\hline Lluvias & 32.5 & 32.5 & 32.5 & 32.5 \\
\hline Nortes & 26.0 & 25.1 & 31.0 & 27.4 \\
\hline Secas & 31.0 & 31.0 & 31.0 & 31.0 \\
\hline \multirow[t]{2}{*}{ Promedio } & 29.8 & 29.5 & 31.5 & \\
\hline & \multicolumn{4}{|c|}{ Profundidad $(\mathrm{cm})$} \\
\hline Lluvias & 158 & 160 & 190 & 169 \\
\hline Nortes & 210 & 170 & 177 & 186 \\
\hline Secas & 130 & 136 & 127 & 131 \\
\hline \multirow[t]{2}{*}{ Promedio } & 166 & 155 & 165 & \\
\hline & \multicolumn{4}{|c|}{ Transparencia $(\mathrm{cm})$} \\
\hline Lluvias & 70 & 70 & 75 & 72 \\
\hline Nortes & 67 & 80 & 30 & 59 \\
\hline Secas & 50 & 90 & 80 & 73 \\
\hline \multirow[t]{2}{*}{ Promedio } & 62 & 80 & 62 & \\
\hline & \multicolumn{4}{|c|}{$\mathrm{pH}$} \\
\hline Lluvias & 7.6 & 7.2 & 6.7 & 7.2 \\
\hline Nortes & 7.8 & 7.6 & 7.7 & 7.7 \\
\hline Secas & 7.4 & 7.3 & 7.4 & 7.4 \\
\hline $\begin{array}{l}\text { Promedio } \\
* * * * * \text { No }\end{array}$ & 7.6 & 7.4 & 7.3 & \\
\hline
\end{tabular}

en el sitio II ( $\mathrm{n}=686), 17$ especies (siete familias y 12 géneros) y en el sitio III $(n=185)$, se registraron 14 especies correspondientes a cinco familias y 11 géneros. Además, se encontraron dos especies exóticas de cíclidos, Oreochromis niloticus representada en los tres sitios, y Parachromis managuense sólo presente en el sitio II. También, se capturaron jóvenes de siluridos representados por la especie Potamarius nelsoni en los sitios I y III durante 
CUADRO 2

Listado de peces de los sitios I, II y III, de los humedales en San Pedro, Balancán

TABLE 2

Fishes checklist in the sites I, II, III at San Pedro wetlands, Balancán

\begin{tabular}{|c|c|c|c|c|c|c|}
\hline Orden & Familia & Especie & I & II & III & Autor y año \\
\hline \multirow[t]{3}{*}{ Siluriformes } & Ariidae & Cathorops aguadulce & $\mathrm{x}$ & $\mathrm{x}$ & $\mathrm{x}$ & (Meek, 1904) \\
\hline & & Potamarius nelsoni & $\mathrm{x}$ & & $\mathrm{x}$ & (Evermann \& Goldsborough, 1902) \\
\hline & Heptapteridae & Rhamdia guatemalensis & $\mathrm{x}$ & & & (Günther, 1864) \\
\hline Batrachoidiformes & Batrachoididae & Batrachoides goldmani & $\mathrm{x}$ & & & (Evermann \& Goldsborough, 1902) \\
\hline \multirow[t]{2}{*}{ Characiformes } & Characidae & Astyanax aeneus & $\mathrm{x}$ & $\mathrm{x}$ & $\mathrm{x}$ & (Günther, 1860) \\
\hline & & Hyphessobrycon compresus & $\mathrm{x}$ & & & (Meek, 1904) \\
\hline \multirow[t]{12}{*}{ Perciformes } & Cichlidae & Amphilophus robertsoni & $\mathrm{x}$ & $\mathrm{x}$ & $\mathrm{x}$ & (Reagan, 1905) \\
\hline & & Oreochromis niloticus * & $\mathrm{x}$ & $\mathrm{x}$ & $\mathrm{x}$ & (Linnaeus, 1758) \\
\hline & & Parachromis managuense $*$ & & $\mathrm{x}$ & $\mathrm{x}$ & (Günther, 1867) \\
\hline & & Petenia splendida & $\mathrm{x}$ & $\mathrm{x}$ & $\mathrm{x}$ & (Günther, 1862) \\
\hline & & Thorichthys affinis & $\mathrm{x}$ & $\mathrm{x}$ & $\mathrm{x}$ & (Günther, 1862) \\
\hline & & Thorichthys helleri & $\mathrm{x}$ & $\mathrm{x}$ & $\mathrm{x}$ & (Steindachner, 1864) \\
\hline & & Thorichthys pasionis & $\mathrm{x}$ & $\mathrm{x}$ & $\mathrm{x}$ & (Rivas, 1962) \\
\hline & & Vieja argentea & $\mathrm{x}$ & & & (Allgayer, 1991) \\
\hline & & Vieja bifasciata & $\mathrm{x}$ & $\mathrm{x}$ & & (Steindachner, 1864) \\
\hline & & Vieja heterospila & $\mathrm{x}$ & $\mathrm{x}$ & $\mathrm{x}$ & (Hubbs, 1936) \\
\hline & & Vieja synspila & $\mathrm{x}$ & $\mathrm{x}$ & $\mathrm{x}$ & (Hubbs, 1935) \\
\hline & Gerreidae & Eugerres mexicanus & $\mathrm{x}$ & $\mathrm{x}$ & $\mathrm{x}$ & (Steindachner, 1863) \\
\hline \multirow[t]{2}{*}{ Clupeiformes } & Clupeidae & Dorosoma anale & $\mathrm{x}$ & & & (Meek, 1904) \\
\hline & & Dorosoma petenense & $\mathrm{x}$ & $\mathrm{x}$ & $\mathrm{x}$ & (Günther, 1867) \\
\hline Lepisosteiformes & Lepisosteidae & Atractosteus tropicus & $\mathrm{x}$ & & & (Gill, 1863) \\
\hline \multirow[t]{4}{*}{ Cyprinidontiformes } & Poeciliidae & Carlhubbsia kidderi & $\mathrm{x}$ & $\mathrm{x}$ & & (Hubbs, 1936) \\
\hline & & Phallichthys fairweatheri & $\mathrm{x}$ & & & (Rosen \& Bailey, 1959) \\
\hline & & Poecilia mexicana & $\mathrm{x}$ & $\mathrm{x}$ & & (Steindachner, 1863) \\
\hline & & Poecilia petenensis & & $\mathrm{x}$ & & (Günther, 1866) \\
\hline
\end{tabular}

Especies exóticas *

la época de secas. Esta especie se encuentra catalogada en peligro de extinción según la NOM-059-ECOL-2001. En cuanto a la riqueza por época, en secas $(n=721)$, se identificaron 19 especies correspondientes a ocho familias y 15 géneros; en la época de lluvias ( $\mathrm{n}=303), 17$ especies (7 familias y 13 géneros) y en nortes $(n=25)$, se registraron cinco especies correspondientes a cuatro familias y tres géneros.

Las familias que presentaron el mayor número de especies fueron Cichlidae con 11 especies: Amphilophus robertsoni, O. niloticus, P. managuense, Petenia splendida, Thorichthys affinis, Thorichthys helleri, Thorichthys pasionis, Vieja argentea, Vieja synspila, Vieja bifasciata y Vieja heterospila, seguida de la familia Poeciliidae con cuatro especies: Carlhubbsia kidderi, Phallichthys fairweatheri, Poecilia mexicana y Poecilia petenensis, mientras que las familias con menor número de especies fueron: Ariidae con dos especies: Cathorops aguadulce, P. nelsoni, Characidae con dos especies: Astyanax aeneus y Hyphessobrycon compressus, la familia Clupeidae con dos especies: Dorosoma anale y Dorosoma petenense, Heptateridae con: Rhamdia guatemalensis, 
Batrachoididae: Batrachoides goldmani, Gerreidae: Eugerres mexicanus y Lepisosteidae: Atractosteus tropicus.

Parámetros ecológicos: El análisis espacial y temporal de la diversidad (H'), riqueza (S) y equidad ( $\mathrm{J}$ '), señaló contrastes entre las comunidades de peces entre los sitios. Los valores más altos de diversidad $\left(H^{\prime}=2.3\right)$ y equidad $\left(\mathrm{J}^{\prime}=0.9\right)$, se presentaron en el sitio III, mientras que la mayor riqueza se dio en el sitio I $(S=4.0)$. Los valores más bajos de H', $S$ y J' fueron registrados en el sitio II (1.6, 2.4, 0.6) (Cuadro 3). El parámetro de riqueza (S) presentó diferencias significativas en los sitios $(\mathrm{F}=5.98, \mathrm{p}<0.05)$. La prueba de Tukey mostró estás diferencias en los sitios I y II (Tukey, $\mathrm{p} \leq 0.05)$. En cuanto a las épocas, la mayor diversidad se presentó en lluvias $\left(\mathrm{H}^{\prime}=2.0\right)$, la mayor riqueza en secas $(S=3.0)$ y la mayor equidad correspondió a nortes $\left(\mathrm{J}^{\prime}=0.8\right)$. Al comparar, los valores de diversidad (H'), riqueza de especies (S) y equidad (J') en las épocas, no se presentaron diferencias significativas

Variación espacial de la densidad y biomasa: La mayor densidad relativa se mostró en el sitio I $\left(0.23 \mathrm{ind} . / \mathrm{m}^{2}\right)$, seguido del sitio II $\left(0.23\right.$ ind.$\left./ \mathrm{m}^{2}\right)$, y por último el sitio III (0.12ind. $/ \mathrm{m}^{2}$ ) (Cuadro 4). Las especies que presentaron mayores densidades fueron: T. affinis $\left(0.160 \mathrm{ind} . / \mathrm{m}^{2}\right)$, T. helleri (0.086ind.$\left./ \mathrm{m}^{2}\right)$ A. aeneus (0.066ind./ $\left.\mathrm{m}^{2}\right)$, P. splendida $\left(0.049 \mathrm{ind} . / \mathrm{m}^{2}\right), P$. petenensis $\left(0.033\right.$ ind.$\left./ \mathrm{m}^{2}\right)$, A. robertsoni $\left(0.013\right.$ ind.$\left./ \mathrm{m}^{2}\right)$, mientras que los valores mínimos estuvieron representados por $D$. petenense, $V$. bifasciata, $B$. goldmani, $R$. guatemalensis y $V$. argentea $\left(>0.001 \mathrm{ind} . / \mathrm{m}^{2}\right)$. En cuanto a biomasa, el valor máximo se presentó en el sitio III $\left(5.34 \mathrm{~g} / \mathrm{m}^{2}\right)$ y el mínimo en el sitio II $\left(3.19 \mathrm{~g} / \mathrm{m}^{2}\right)$. En relación con las especies, éstas estuvieron representadas por P. splendida y A. tropicus, las cuales exhibieron los valores más altos $\left(2.78 \mathrm{~g} / \mathrm{m}^{2}\right.$, $\left.2.049 \mathrm{~g} / \mathrm{m}^{2}\right)$, mientras que los valores mínimos estuvieron representados por $D$. anale y $H$. compresus, con valores de $\left(<0.001 \mathrm{~g} / \mathrm{m}^{2}\right)$. La densidad y la biomasa no presentaron diferencias significativas entre sitios.

Variación temporal de la densidad y biomasa: La mayor densidad se presentó en la época de secas $\left(0.43 \mathrm{ind} . / \mathrm{m}^{2}\right)$, seguida de lluvias $\left(0.21\right.$ ind.$\left./ \mathrm{m}^{2}\right)$ y por último en nortes $\left(0.02\right.$ ind.$\left./ \mathrm{m}^{2}\right)$. No se presentaron diferencias significativas entre las épocas. El diagrama de Olmstead-Tukey mostró cinco especies dominantes en densidad, $P$. splendida, $V$. heterospila, $V$. synspila, D. petenense, A. aeneus, ésta última fue la que exhibió el valor más alto $\left(0.06\right.$ ind.$\left./ \mathrm{m}^{2}\right)$, así mismo, se registraron cinco especies constantes, en donde $T$. affinis

\section{CUADRO 3}

Parámetros ecológicos de la comunidad de peces en las épocas y sitios de los humedales de San Pedro $\mathrm{H}^{\prime}=$ diversidad (bits. ind. $\mathrm{m}^{2}$ ), $\mathrm{S}=$ riqueza, $\mathrm{J}=$ equidad

TABLE 3

Ecological parameters of fish community at the sites and seasons at San Pedro wetlands $\mathrm{H}^{\prime}=$ diversity (bits. ind./ $\mathrm{m}^{2}$ ), $\mathrm{S}=$ richness, $\mathrm{J}=$ equitability

\begin{tabular}{lcccccc} 
& & Espacial & & \multicolumn{3}{c}{ Épocas } \\
& I & II & III & Secas & Lluvias & Nortes \\
No. de taxones & 23 & 17 & 14 & 21 & 17 & 5 \\
No. de individuos & 178 & 686 & 185 & 721 & 303 & 25 \\
Shannon $\left(\mathrm{H}^{\prime}\right)$ & 2.1 & 1.6 & 2.3 & 1.6 & 2.0 & 1.3 \\
Riqueza $(\mathrm{S})$ & $4.0^{* *}$ & $2.4 *$ & 2.5 & 3.0 & 2.8 & 1.2 \\
Equidad $(\mathrm{J})$ & 0.7 & 0.6 & 0.9 & 0.5 & 0.7 & 0.8 \\
$*$ p $\leq 0.05$ & & & & & &
\end{tabular}


CUADRO 4

Distribución espacial de la densidad (ind. $\left./ \mathrm{m}^{2}\right)$ y biomasa $\left(\mathrm{g} / \mathrm{m}^{2}\right)$ de los peces en los humedales del río San Pedro

TABLE 4

Fish spatial distribution of density (ind. $\left./ \mathrm{m}^{2}\right)$ and biomass $\left(\mathrm{g} / \mathrm{m}^{2}\right)$ at San Pedro wetlands Balancán, Tabasco

\begin{tabular}{|c|c|c|c|c|c|c|c|c|c|}
\hline \multirow{2}{*}{ Especie } & \multicolumn{4}{|c|}{ Densidad (ind. $/ \mathrm{m}^{2}$ ) } & \multirow{2}{*}{ Especie } & \multicolumn{3}{|c|}{ Biomasa $\left(\mathrm{g} / \mathrm{m}^{2}\right)$} & \multirow{2}{*}{ Total } \\
\hline & I & II & III & Total & & I & II & III & \\
\hline Thorichthys affinis & 0.1026 & 0.0064 & 0.0513 & 0.1603 & Petenia splendida & 0.5031 & 0.8551 & 1.4265 & 2.7847 \\
\hline Thorichthys helleri & 0.0071 & 0.0691 & 0.0100 & 0.0862 & Atractosteus tropicus & 1.6909 & - & 0.3583 & 2.0491 \\
\hline Astyanax aeneus & 0.0192 & 0.0249 & 0.0221 & 0.0662 & Parachromis managuense & 0.2927 & 0.0370 & 0.9701 & 1.2998 \\
\hline Petenia splendida & 0.0135 & 0.0342 & 0.0021 & 0.0498 & Vieja heterospila & 0.1571 & 0.1692 & 0.8733 & 1.1996 \\
\hline Poecilia petenensis & - & 0.0334 & - & 0.0334 & Oreochromis niloticus & 0.1698 & 0.4255 & 0.5625 & 1.1578 \\
\hline Vieja synspila & 0.0150 & 0.0142 & 0.0028 & 0.0320 & Thorichthys affinis & 0.2367 & 0.7053 & 0.1514 & 1.0934 \\
\hline Vieja heterospila & 0.0249 & 0.0028 & 0.0021 & 0.0298 & Potamarius nelsoni & 0.3917 & - & 0.2137 & 0.6054 \\
\hline Dorosoma petenense & 0.0036 & 0.0064 & 0.0121 & 0.0221 & Vieja synspila & 0.0511 & 0.2115 & 0.1647 & 0.4274 \\
\hline Thorichthys pasionis & 0.0078 & 0.0128 & - & 0.0207 & Thorichthys helleri & 0.0719 & 0.2830 & 0.0542 & 0.4090 \\
\hline Oreochromis niloticus & 0.0064 & 0.0107 & 0.0014 & 0.0185 & Dorosoma petenense & 0.2427 & 0.015 & 0.1413 & 0.3985 \\
\hline Amphilophus robertsoni & 0.0043 & 0.0064 & 0.0028 & 0.0135 & Amphilophus robertsoni & 0.0183 & 0.2375 & 0.1026 & 0.3585 \\
\hline Eugerres mexicanus & 0.0085 & 0.0014 & 0.0028 & 0.0128 & Thorichthys pasionis & 0.0149 & 0.1345 & 0.0960 & 0.2305 \\
\hline Phallichthys fairweatheri & 0.0085 & - & 0.0021 & 0.0106 & Astyanax aeneus & 0.0368 & 0.0620 & 0.0543 & 0.1531 \\
\hline Cathorops aguadulce & 0.0078 & - & 0.0007 & 0.0085 & Cathorops aguadulce & 0.0073 & - & 0.1218 & 0.1291 \\
\hline Carlhubbsia kidderi & - & 0.0036 & 0.0028 & 0.0064 & Phallichthys fairweatheri & 0.0149 & - & 0.0415 & 0.0563 \\
\hline Parachromis managuense & 0.0036 & 0.0007 & 0.0014 & 0.0057 & Eugerres mexicanus & 0.0286 & 0.0051 & 0.0175 & 0.0511 \\
\hline Poecilia mexicana & - & 0.0050 & - & 0.0050 & Batrachoides goldmani & 0.0462 & - & - & 0.0462 \\
\hline Actractosteus tropicus & 0.0007 & - & 0.0021 & 0.0028 & Poecilia mexicana & 0.0149 & 0.0363 & - & 0.0363 \\
\hline Hyphessobrycon compressus & - & - & 0.0028 & 0.0028 & Vieja argentea & 0.0181 & - & - & 0.0181 \\
\hline Potamarius nelsoni & 0.0007 & - & 0.0014 & 0.0021 & Carlhubbsia kidderi & 0.0060 & 0.0052 & - & 0.0112 \\
\hline Dorosoma anale & - & - & 0.0014 & 0.0014 & Vieja bifasciata & 0.0035 & 0.0043 & - & 0.0078 \\
\hline Vieja bifasciata & - & 0.0007 & 0.0007 & 0.0014 & Rhamdia guatemalensis & 0.0078 & - & - & 0.0078 \\
\hline Batrachoides goldmani & - & - & 0.0007 & 0.0007 & Poecilia petenensis & 0.0149 & 0.0043 & - & 0.0043 \\
\hline Rhamdia guatemalensis & - & - & 0.0007 & 0.0007 & Hyphessobrycon compresus & 0.0008 & - & - & 0.0008 \\
\hline Vieja argentea & - & - & 0.0007 & 0.0007 & Dorosoma anale & 0.0005 & - & - & 0.0005 \\
\hline Total & 0.2342 & 0.2327 & 0.1273 & 0.5942 & & 4.0409 & 3.1903 & 5.3495 & 12.5360 \\
\hline
\end{tabular}

fue la que registró el mayor valor (0.27ind./ $\mathrm{m}^{2}$ ) y un total de 15 especies fueron consideradas como ocasionales, y se observó a la especie $A$. robertsoni con el valor máximo $\left(0.01\right.$ ind.$\left./ \mathrm{m}^{2}\right)$. En la zona de los humedales del río San Pedro, las fluctuaciones en el nivel del agua está directamente relacionados con el régimen de precipitación pluvial en las épocas de secas y lluvias, que determinan cambios en las abundancias de las diferentes especies presentes en estos hábitats, un ejemplo de ello fue la especie $P$. splendida la cual disminuye su densidad en la época de nortes. Las densidades más altas se encontraron en las épocas de secas y lluvias, cuando los sitios presentan menores profundidades.

La mayor biomasa se registró en la época de secas $\left(8.30 \mathrm{~g} / \mathrm{m}^{2}\right)$, seguida de lluvias $\left(2.16 \mathrm{~g} / \mathrm{m}^{2}\right)$, y por último en nortes $\left(0.03 \mathrm{~g} / \mathrm{m}^{2}\right)$. Entonces, se presentaron diferencias significativas en las épocas, donde se observaron estas diferencias entre las épocas de secas y lluvias, así como 
en nortes y secas (Tukey, p<0.05) (Cuadro 5). El diagrama de Olmstead-Tukey determinó un total de cinco especies dominantes en biomasa (P. splendida, V. heterospila, T. affinis, O. niloticus, $P$. managuense), siendo $P$. splendida la que registró el máximo valor $\left(2.06 \mathrm{~g} / \mathrm{m}^{2}\right)$; seis especies fueron constantes, de las cuales $T$. pasionis obtuvo el valor más alto $\left(0.23 \mathrm{~g} / \mathrm{m}^{2}\right)$, asimismo, se observaron dos especies frecuentes ambas con altos valores $(P$. nelsoni $0.60 \mathrm{~g} / \mathrm{m}^{2}$ y A. tropicus $0.49 \mathrm{~g} / \mathrm{m}^{2}$ ), finalmente
12 especies fueron ocasionales y A.robertsoni la que exhibió el mayor valor $\left(0.35 \mathrm{~g} / \mathrm{m}^{2}\right)$.

Análisis temporal de la estructura de tallas: Las longitudes mayores de la estructura de tallas estuvieron representadas por: $A$. tropicus $(\mathrm{n}=1 ; 510 \mathrm{~mm})$, P. splendida $(\mathrm{n}=60$, $135.8 \mathrm{~mm}), O$. niloticus $(\mathrm{n}=23,125 \mathrm{~mm}), C$. aguadulce $(\mathrm{n}=8,121.1 \mathrm{~mm}), R$. guatemalensis $(\mathrm{n}=1,119.2 \mathrm{~mm})$ en la época de secas; $B$. goldmani $(\mathrm{n}=1,171.1 \mathrm{~mm})$, P. splendida $(\mathrm{n}=6$,

\section{CUADRO 5}

Distribución temporal de la densidad y biomasa de peces de los humedales del río San Pedro ( $\mathrm{D}=$ especie dominante, $\mathrm{C}=$ especie constante, $\mathrm{F}=$ especie frecuente y $\mathrm{O}=$ especie ocasional o rara)

TABLE 5

Seasons distribution of the fishes density and biomass at San Pedro wetlands

( $\mathrm{D}=$ dominant species, $\mathrm{C}=$ constant species, $\mathrm{F}=$ frequent species and $\mathrm{O}=$ occasional or rare species)

\begin{tabular}{|c|c|c|c|c|c|c|c|c|c|c|}
\hline \multirow{2}{*}{ Especie/ época } & \multicolumn{5}{|c|}{ Densidad (ind. $/ \mathrm{m}^{2}$ ) } & \multicolumn{5}{|c|}{ Biomasa $\left(\mathrm{g} / \mathrm{m}^{2}\right)$} \\
\hline & Secas & Lluvias & Nortes & Total & Categoría & Secas & Lluvias & Nortes & Total & Categoría \\
\hline Petenia splendida & 0.0427 & 0.0043 & - & 0.0470 & D & 1.6425 & 0.4185 & - & 2.0610 & D \\
\hline Vieja heterospila & 0.0192 & 0.0114 & - & 0.0306 & $\mathrm{D}$ & 1.5046 & 0.3917 & - & 1.8963 & $\mathrm{D}$ \\
\hline Thorichthys affinis & 0.2407 & 0.0306 & 0.0021 & 0.2735 & $\mathrm{C}$ & 0.9270 & 0.1512 & 0.0023 & 1.0805 & $\mathrm{D}$ \\
\hline Oreochromis niloticus & 0.0164 & 0.0021 & - & 0.0185 & $\mathrm{C}$ & 0.8528 & 0.3049 & - & 1.1578 & $\mathrm{D}$ \\
\hline Parachromis managuense & 0.0028 & 0.0007 & - & 0.0036 & $\mathrm{O}$ & 0.7835 & 0.0370 & - & 0.8204 & $\mathrm{D}$ \\
\hline Thorichthys helleri & 0.0064 & 0.0783 & 0.0075 & 0.0923 & $\mathrm{C}$ & 0.0677 & 0.3366 & 0.0075 & 0.4043 & $\mathrm{C}$ \\
\hline Vieja synspila & 0.0228 & 0.0100 & - & 0.0328 & $\mathrm{D}$ & 0.2695 & 0.1659 & - & 0.4354 & $\mathrm{C}$ \\
\hline Dorosoma petenense & 0.0114 & 0.0078 & 0.0100 & 0.0292 & $\mathrm{D}$ & 0.3573 & 0.0390 & 0.0091 & 0.4054 & $\mathrm{C}$ \\
\hline Potamarius nelsoni & 0.0014 & - & - & 0.0014 & $\mathrm{O}$ & 0.6054 & - & - & 0.6054 & $\mathrm{~F}$ \\
\hline Atractosteus tropicus & 0.0021 & - & - & 0.0021 & $\mathrm{O}$ & 0.4986 & - & - & 0.4986 & $\mathrm{~F}$ \\
\hline Thorichthys pasionis & 0.0128 & 0.0093 & 0.0008 & 0.0229 & $\mathrm{C}$ & 0.1667 & 0.0688 & 0.0078 & 0.2355 & $\mathrm{C}$ \\
\hline Astyanax aeneus & 0.0264 & 0.0370 & 0.0028 & 0.0662 & $\mathrm{D}$ & 0.0662 & 0.0838 & 0.0031 & 0.1531 & $\mathrm{C}$ \\
\hline Amphilophus robertsoni & 0.0135 & - & - & 0.0135 & $\mathrm{O}$ & 0.3585 & - & - & 0.3585 & $\mathrm{O}$ \\
\hline Cathorops aguadulce & 0.0000 & 0.0028 & - & 0.0028 & $\mathrm{O}$ & 0.0890 & 0.0397 & - & 0.1287 & $\mathrm{O}$ \\
\hline Eugerres mexicanus & 0.0014 & 0.0043 & - & 0.0057 & $\mathrm{C}$ & 0.0100 & 0.0336 & - & 0.0436 & $\mathrm{O}$ \\
\hline Carlhubbsia kidderi & 0.0057 & 0.0007 & - & 0.0064 & $\mathrm{O}$ & 0.0105 & 0.0007 & - & 0.0112 & $\mathrm{O}$ \\
\hline Poecilia mexicana & 0.0036 & - & - & 0.0036 & $\mathrm{O}$ & 0.0231 & - & - & 0.0231 & $\mathrm{O}$ \\
\hline Vieja bifasciata & 0.0014 & - & - & 0.0014 & $\mathrm{O}$ & 0.0078 & - & - & 0.0078 & $\mathrm{O}$ \\
\hline Phallichthys fairweatheri & - & 0.0085 & - & 0.0085 & $\mathrm{O}$ & - & 0.0418 & - & 0.0418 & $\mathrm{O}$ \\
\hline Batrachoides goldmani & - & 0.0007 & - & 0.0007 & $\mathrm{O}$ & - & 0.0462 & - & 0.0462 & $\mathrm{O}$ \\
\hline Poecilia petenensis & 0.0043 & - & - & 0.0043 & $\mathrm{O}$ & 0.0334 & - & - & 0.0377 & $\mathrm{C}$ \\
\hline Vieja argentea & 0.0007 & - & - & 0.0007 & $\mathrm{O}$ & 0.0181 & - & - & 0.0181 & $\mathrm{O}$ \\
\hline Rhamdia guatemalensis & 0.0007 & - & - & 0.0007 & $\mathrm{O}$ & 0.0078 & - & - & 0.0078 & $\mathrm{O}$ \\
\hline Hyphessobrycon compressus & - & 0.0028 & - & 0.0028 & $\mathrm{O}$ & - & 0.0008 & - & 0.0008 & $\mathrm{O}$ \\
\hline Dorosoma anale & - & 0.0014 & - & 0.0014 & $\mathrm{O}$ & - & 0.0005 & - & 0.0005 & $\mathrm{O}$ \\
\hline Total & 0.4366 & 0.2130 & 0.0233 & 0.6729 & & 8.300 & 2.165 & 0.030 & 10.479 & \\
\hline
\end{tabular}


$150.6 \mathrm{~mm}), P$. managuense $(\mathrm{n}=1,138.8 \mathrm{~mm}), O$. niloticus $(\mathrm{n}=3,137.8 \mathrm{~mm})$, C. aguadulce $(\mathrm{n}=4$, $117.9 \mathrm{~mm}$ ) en la época de lluvias y finalmente, especies de cuerpos pequeños representados por: T. helleri $(\mathrm{n}=3,57.1 \mathrm{~mm})$, D. petenense $(\mathrm{n}=14,48.8 \mathrm{~mm})$, A. aeneus $(\mathrm{n}=4,42.9 \mathrm{~mm})$, T. pasionis $(\mathrm{n}=1,41.3 \mathrm{~mm})$ у T. affinis $(\mathrm{n}=3$, $40.9 \mathrm{~mm}$ ), en la época de nortes (Cuadro 6).

El análisis de correspondencia canónica entre las asociaciones de peces y las variables ambientales en las épocas de secas, lluvias y nortes versus especies (Fig. 2): C. aguadulce, $P$. nelsoni, R. guatemalensis, B. goldmani, A. aeneus, $H$. compresus, A. robertsoni, $O$. niloticus, $P$. managuense, $P$. splendida, $T$. affinis, $T$. helleri, T. pasionis, V. argentea, V. bifasciata, $V$. heterospila, V. synspila, E. mexicanus, D. anale, D. petenense, A. tropicus, C. kidderi, $P$. fairweatheri, $P$. mexicana, $P$. petenensis, explicó el $68.7 \%$ en el primer eje y el $31.3 \%$ en el segundo, y produce un total de $100 \%$ de la variación explicada en estos componentes.
Las variables continuas que presentaron mayor asociación con respecto a la distribución de la comunidad de peces por épocas fueron: profundidad, $\mathrm{pH}$ y oxígeno disuelto. El primer eje, se interpreta principalmente, con la contraposición de época de secas y lluvias. La época de secas se encuentra asociada principalmente con alta transparencia, mientras que las épocas de lluvias y nortes se encuentran asociadas a temperatura y profundidad. Lo anterior, promueve menor temperatura y mayor profundidad entre las épocas, lo cual genera una disimilitud de especies entre nortes y lluvias.

El análisis de conglomerados identificó tres grupos afines. El primer grupo se caracterizó por la alta frecuencia y densidad de T. affinis, $P$. splendida, $O$. niloticus, A. robertsoni, $P$. nelsoni, P. mexicana, P. petenensis, C. kidderi, $R$. guatemalensis y A.tropicus, que mostraron una alta asociación con la transparencia, oxígeno disuelto y $\mathrm{pH}$. Por su parte, las especies de $D$. petenense, A. aeneus, $P$. managuense, $D$.

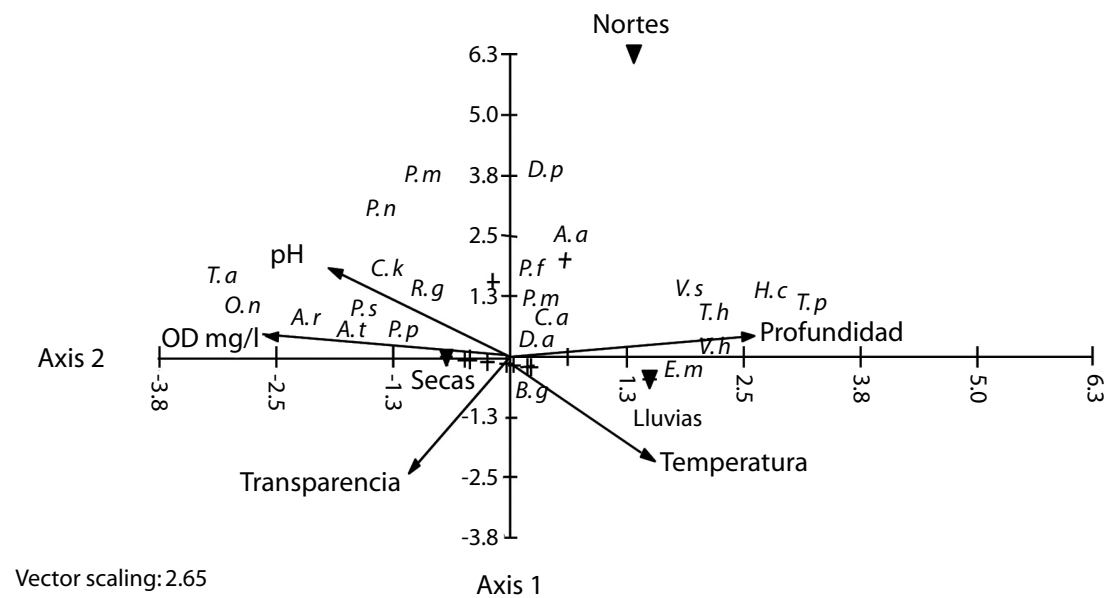

Fig. 2. Análisis de correspondencia canónica entre las asociaciones de peces y las variables ambientales en las épocas de Secas, Lluvias y Nortes. Especies: C. aguadulce, P. nelsoni, R. guatemalensis, B. goldmani, A. aeneus, H. compresus, A. robertsoni, O. niloticus, P. managuense, P. splendida, T. affinis, T. helleri, T. pasionis, V. argentea, V. bifasciata, V. heterospila, V. synspila, E. mexicanus, D. anale, D. petenense, A. tropicus, C. kidderi, P. fairweatheri, P. mexicana y P. petenensis.

Fig. 2. Canonic correspondence analysis between fish and environmental variables during the dry, rainy and cold seasons. Species: C. aguadulce, P. nelsoni, R. guatemalensis, B. goldmani, A. aeneus, H. compresus, A. robertsoni, O. niloticus, P. managuense, P. splendida, T. affinis, T. helleri, T. pasionis, V. argentea, V. bifasciata, V. heterospila, V. synspila, E. mexicanus, D. anale, D. petenense, A. tropicus, C. kidderi, P. fairweatheri, P. mexicana y P. petenensis. 


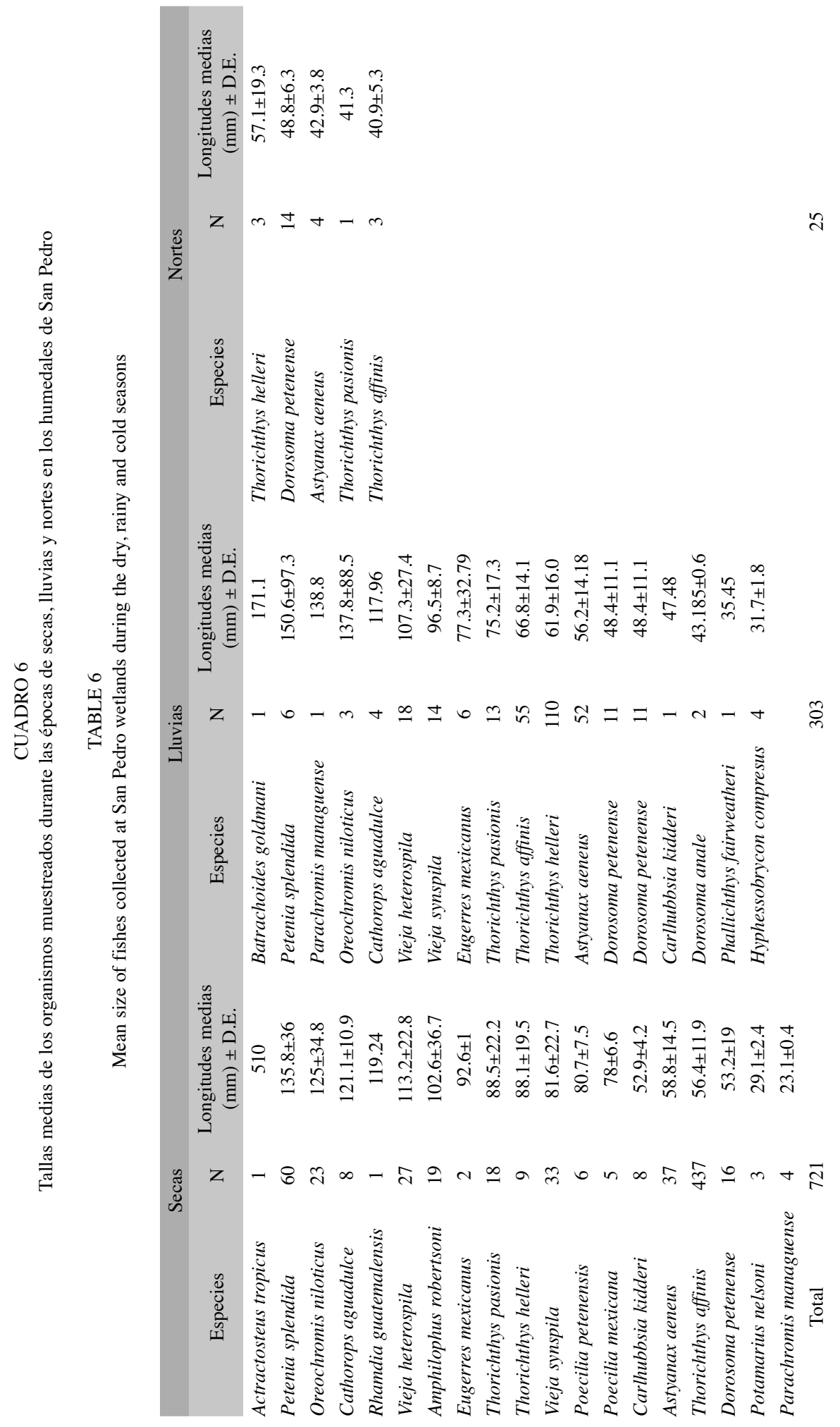


anale, P. fairweatheri, H. compresus, T. helleri, $T$. pasionis, $V$. argentea, $V$. bifasciata y $V$. heterospila, se encontraron asociadas a la profundidad en la época de nortes; mientras que $E$. mexicanus, $C$. aguadulce y B. goldmani, a las lluvias definidas por la temperatura.

\section{DISCUSIÓN}

Componente ambiental: Diversos estudios han señalado la influencia de las variables ambientales en la composición de las asociaciones de peces (Gelwick et al. 2001, Hoeinghaus et al. 2003, Kobza et al. 2004). Otros autores atribuyen que estas variaciones en la estructura de las comunidades de peces son causadas por las diversas interacciones biológicas, como la cobertura de macrófitas, la complejidad del hábitat, las redes tróficas y las relaciones de competencia y depredación (Kupschus \& Tremain 2001, Heredia 2002, Petry et al. 2003).

En los humedales del río San Pedro, la variabilidad de los parámetros físico-químicos está relacionada principalmente por las condiciones hidrológicas determinadas por las épocas, es decir, lluvias, nortes y secas. Los cambios en el nivel del agua se encuentran asociados por el régimen de precipitación pluvial, y se establece menor nivel de agua en los sitios estudiados en la época de secas; sin embargo, los niveles máximos encontrados en estos ecosistemas fue en la época de nortes, esto debido a su máximo incremento del caudal por la precipitación a finales de la época de lluvias generado en los meses de septiembre-octubre, que son los meses más lluviosos en la zona.

Por su parte, el oxígeno disuelto es un factor fundamental en la dinámica acuática, la abundancia o escases de este parámetro limita o favorece la presencia y/o ausencia de organismos, afectando el crecimiento, sobrevivencia y la reproducción (Quiroz et al. 2010). Las variaciones en su concentración en los humedales pueden deberse a la temperatura del agua, a la aportación de sedimentos alóctonos derivado del flujo de inundación estacional o a la generación de oxígeno dentro del cuerpo de agua por la actividad de organismos fotosintéticos. $\mathrm{La}$ temperatura del agua está influenciada por las condiciones ambientales de la zona y la incidencia de luz solar, ya que la energía luminosa es absorbida exponencialmente con respecto a la profundidad y la mayor parte del calor es retenido en la capa superior del sistema (Quiroz et al. 2010). La variación de la temperatura coincidió en las épocas, donde la época de lluvias es la más cálida, esto podría ser causado por una canícula o sequía intraestival que va de junio a septiembre en la región (Pereyra-Díaz et al. 2004). No obstante, la relación inversa entre temperatura y la concentración de oxígeno disuelto puede verse alterada en estos ambientes naturales por efecto de los procesos de fotosíntesis y respiración (Graham 1995).

Los humedales del río San Pedro presentan características hidrológicas similares a los Petenes en Campeche, donde Torres-Castro et al. (2008), observaron que la comunidad de peces se adaptan a las fluctuaciones del nivel del agua, tanto estacionales como interanuales. También en este estudio las condiciones hidrológicas durante las épocas determinaron los cambios en las densidades, biomasa, abundancias y riqueza de las especies.

Las diferentes especies de peces que habi$\tan$ los humedales tropicales presentan un amplio repertorio de estrategias de vida (longitud mínima a la maduración, periodo de vida corto, épocas de desove parcial, alta fecundidad de huevos a la alta disponibilidad de alimento) cuyo conocimiento no sólo permite explicar patrones de comportamiento, sino enriquecer modelos ecológicos y efectuar predicciones sobre la dinámica comunitaria y del sistema (Winemiller \& Jepsen 1998).

Composición y diversidad: El número de familias y especies registradas en este estudio fue menor a las reportadas por Chávez et al. (1988) y Noiset \& Hernández (1991) para el río San Pedro y su zona de inundación. La tolerancia a las variaciones ambientales y la plasticidad genética que caracteriza a la familia Cichlidae (Urriola et al. 2004, Peña-Mendoza et al. 2005, Sáenz et al. 2006), influye en este taxón y por lo tanto es el mejor representado, 
estos atributos le confieren una mejor posición sobre otros componentes de la ictiofauna en la zona de los humedales. La condición dominante de esta familia, también fue reportada por Valdez-Moreno et al. 2005, donde mencionan que las familias Cichlidae y Poeciliidae fueron las más diversas en dos regiones importantes de los departamentos del Petén y la Alta Verapaz en Guatemala.

La dominancia de las especies estuvo representada por $A$. aeneus y $P$. splendida caso contrario con lo reportado por Noiset \& Hernández (1991), donde mencionan que ésta estuvo representada por "Cichlasoma" synspilum. Especie que según Espinosa-Pérez \& Daza-Zepeda (2005), en su revisión la registraron como "Cichlasoma" (Theraps) synspilum para la cuenca Grijalva-Usumacinta en el estado de Tabasco. La amplia diversificación regional que presenta el género Thorichthys, así como, algunos aspectos reproductivos (longitud mínima a la maduración $50 \mathrm{~mm}$ ), a través del año y con características de agrupación en grandes concentraciones, pueden explicar la alta densidad encontrada en la zona (Rodiles-Hernández \& González-Díaz 2006).

Las mayores tallas se capturaron en la época secas, lo cual sugiere un desplazamiento lateral hacia la zona de inundación por las especies adultas como el caso de A. tropicus, el cual realiza desplazamiento reproductivo en busca de zonas someras o poco profundas con vegetación acuática para su apareamiento y desove, y se realiza este comportamiento reproductivo a finales de la época de lluvias (agostoseptiembre) (Chávez et al. 1988). Como patrón general se encontró que la época con mejor calidad ambiental y mayor disponibilidad de alimento fue secas, esto debido al incremento de la temperatura, la cual permite incrementar el desarrollo de organismos autótrofos principalmente, lo que permitió un incremento en riqueza de especies.

Los valores de diversidad (H') obtenidos en este estudio, fueron menores a los estimados por Barrientos (1999), para varios taxones de la comunidad de peces en la parte alta de los ríos San Pedro y Sacluc en un área de influencia del Petén, Guatemala. La baja riqueza en la época de nortes, probablemente se debió al patrón de migración que las especies presentes en estos ambientes realizan en busca de zonas con mayor vegetación acuática y abundancia de alimento.

La presencia de dos especies exóticas o invasoras como fue el caso, $O$. niloticus (tilapia) y P. managuense (pinta), en los humedales se debió con fines de repoblamiento en la cuenca Usumacinta y a su dispersión a través de los pulsos de inundación, según los primeros reportes en los años 1987 (Noiset \& Hernández 1991). Lo anterior ha generado una serie de controversias, acerca de sí realmente estas especies en particular ocasionan algún tipo de impacto ecológico sobre las especies nativas en la zona, que hasta el momento se desconoce por completo el efecto que estén presentando; sin embargo, la pesquería de las tilapias se considera ya importante en la captura comercial, por lo que se deberían tomar precauciones al respecto (Espinosa-Pérez \& Daza-Zepeda 2005).

Relación especie-épocas: La estacionalidad afecta ciertas especies pero no afecta la composición de las comunidades de peces. Tal como lo indicó Matthews (1998), los patrones de comportamiento incluyen desde movimientos diarios o por épocas o migraciones a largas distancias, el cual es representado por un comportamiento natural (Suárez et al. 2004). En los humedales del río San Pedro algunas especies aumentan sus frecuencias y densidades, las cuales les funcionan como estrategias para tolerar las fluctuaciones en las concentraciones de oxígeno disuelto y la transparencia que frecuentemente sucede en estos ecosistemas determinados por la época de secas. El segundo grupo estuvo asociado con el parámetro de profundidad en nortes, y el último grupo con mayor afinidad a la temperatura influenciado por la época de lluvias.

\section{AGRADECIMIENTOS}

Al financiamiento otorgado por Fondos Mixtos del Consejo Nacional de Ciencia y 
Tecnología -Gobierno del Estado de Tabascoal proyecto $\mathrm{TAB}-\mathrm{C} 006-\mathrm{CO} 2-34685$ "Inventario de las microcuencas del municipio de Balancán, Tabasco", a Alfonso González Díaz, por su asesoría en la identificación de las especies en el laboratorio de Ictiología de la Unidad San Cristóbal de Ecosur. A Rodimiro Ramos Reyes encargado del laboratorio del Sistema de Información Geográfica de la Unidad Villahermosa, por el apoyo en la elaboración de la figura del área de estudio, y a la Sociedad Cooperativa de Pescadores "Ribereños del San Pedro", por brindar las facilidades y apoyo con sus equipos de trabajo, mismas que fueron necesarias para la realización de este trabajo.

\section{RESUMEN}

Los humedales de San Pedro sostienen redes tróficas del sistema fluvial debido a la alta disponibilidad de hábitats y a sus variaciones espacio-temporales. En esta investigación se estudió la composición, densidad y biomasa de la ictiofauna de los humedales; se capturaron 1049 organismos de 25 especies, dos son exóticas: Oreochromis niloticus y Parachromis managuense. Con 23 especies capturadas en el sitio I, 17 en el II y 14 en el III. En la época de secas se obtuvo mayor riqueza con 21 especies, lluvias con 17 y cinco en nortes. La mayor densidad se mostró en el sitio II $\left(0.23\right.$ ind.$\left./ \mathrm{m}^{2}\right)$, seguido de los sitios I y III $(0.12$, $\left.0.12 \mathrm{ind} . / \mathrm{m}^{2}\right)$. La mayor biomasa fue en secas $\left(8.30 \mathrm{~g} / \mathrm{m}^{2}\right)$, seguida de lluvias $\left(2.1 \mathrm{~g} / \mathrm{m}^{2}\right)$ y por último nortes $\left(0.01 \mathrm{~g} / \mathrm{m}^{2}\right)$. La mayor densidad se mostró en secas $\left(0.43 i n d . / \mathrm{m}^{2}\right)$ y la mínima en nortes $\left(0.023\right.$ ind.$\left./ \mathrm{m}^{2}\right)$. Las especies dominantes en densidad según el diagrama Olmstead-Tukey fueron: Petenia splendida, Vieja heterospila, Vieja synspila, Dorosoma petenense y Astyanax aeneus. Por lo tanto, existieron diferencias significativas en los parámetros ecológicos y ambientales. Al considerar la variación en la riqueza podemos concluir que los humedales del río San Pedro, es un sistema donde la composición de la ictiofauna responde a las fluctuaciones espaciales y temporales.

Palabras clave: biomasa, densidad, dominancia, riqueza específica, humedales del río San Pedro.

\section{REFERENCIAS}

Álvarez, J. 1970. Peces Mexicanos (claves). Secretaría de Industria y Comercio, México.

Anónimo. 1999. Definición de humedales y sistema de clasificación de tipos de humedales de la Convención de Ramsar (Consultado: 23 marzo 2007, http://www. ramsar.org/ris/key_ris_types_s.htm).

Anónimo. 2002. Regiones hidrológicas prioritarias. Comisión nacional para el conocimiento y uso de la biodiversidad. México. Regiones hidrológicas prioritarias 90, 91 y 92. (Consultado: 19 de junio 207, http:// www.conabio.gob.mx).

Barba, M.E., J.R. Mendoza \& R.R. Reyes. 2006. Clasificación de los humedales de Tabasco mediante sistemas de información geográfica. Univ. Cienc., Univ. Juárez. Autón. Tabasco 23: 101-110.

Barrientos, C.C. 1999. Caracterización de la ictiofauna con importancia alimenticia de los ríos San Pedro y Sacluc en el área de influencia de la estación Biológica las Guacamayas. Tesis de licenciatura, Universidad de San Carlos, el Petén, Guatemala.

Chávez, L.M., A.E. Mattews \& M.H. Pérez. 1988. Biología de los peces del río San Pedro en vistas a determinar su potencial para la piscicultura. INIREB, Xalapa, Veracruz, México.

Cowardin, L.M., V. Carter, F.C. Golet \& E.T. Laroe. 1979. Classification of wetlands and deepwater habitats of the United States. Dep. Interior, Fish and Wildlife. Serv., EEUU.

Daniel, W. 2008. Bioestadística: Base para el análisis de las ciencias de la salud. Limusa Wiley, México. D.F., México.

Dunnett, C. 1980. Pairwise multiple comparisons in the unequal variance case. J. Am. Stat. Assoc. 75: 796-800.

Espinosa-Pérez, H. \& A. Daza-Zepeda. 2005. Peces, p. 225-240. In J. Bueno, F. Álvarez \& S. Santiago (eds.). Biodiversidad del estado de Tabasco. Instituto de Biología, Universidad Nacional Autónoma de México, Comisión Nacional de Biodiversidad, México. D.F., México.

Estrada, L.F. 2008. Inventario de humedales del municipio de Balancán, Tabasco: Biomonitoreo de ecosistemas lénticos y loticos. Tesis de Licenciatura, Universidad Juárez Autónoma de Tabasco, México.

Gelwick, F.P., S. Akin, D.A. Arrington \& K.O. Winemiller. 2001. Fish assemblage structure in relation to environmental variation in a Texas Gulf coastal wetland. Estuaries 24: 285-296.

Graham, J.B. 1995. Air-breathing fishes: evolution, diversity and adaptation. Academic, Londres, Inglaterra.

Heredia, M. 2002. Contribución al conocimiento taxonómico y ecológico de la familia Cichlidae: Ictiofauna 
de la Reserva de la Biósfera de Calakmul, Campeche. Tesis de Licenciatura, Universidad Autónoma Metropolitana, México D.F., México.

Hoeinghaus, D.J., C.A. Layman, D. Albrey \& K.O. Winemiller. 2003. Spatiotemporal variation in fish assemblage structure in tropical floodplain creeks. Env. Biol. Fishes 67: 379-387.

INEGI. 2006. Anuario estadístico municipal: Balancán. Instituto Nacional de Estadística Geografía e Informática, Tabasco, México.

Junk, W.J. \& R.L. Welcome. 1990. Floodplains, p. 491-524. In B.C. Patten (eds.). Wetlands and shallow continental water bodies. SPB Academic, La Haya, Holanda.

Kent, D.M. 2000. Applied wetlands science and technology. Lewis, Florida, EEUU.

Kobza, R.M., J.C. Trexler, W.F. Loftus \& S.A. Perry. 2004. Community structure of fishes inhabiting aquatic refuges in a threatened karst wetland and its implications for ecosystem management. Biol. Conserv. 116: $153-165$.

Kupschus, S. \& D. Tremain. 2001. Associations between fish assemblages and environmental factors in nearshore habitats of a subtropical estuary. J. Fish Biol. 58: 1383-1403.

Levene, H. 1960. Robust test for equality on variance, $\mathrm{p}$. 278-292. In I. Olkin, S.G. Ghurye, W. Hoeffding, W.G. Madow \& H.B. Mann (eds.). Contributions to probability and statics. Stanford University, California, EEUU.

Macossay, C.A. 2008. Ictiofauna del humedal tropical Pantanos de Centla al Sur del Golfo de México. Tesis de Licenciatura, Universidad Juárez Autónoma de Tabasco, México.

Margalef, R. 1969. Perspectives in ecologycal theory. Universidad de Chicago, Chicago, Illinois, EEUU.

Matthews, W.J. 1998. Patterns in freshwater fish ecology. Chapman \& Hall, Nueva York, EEUU.

Methven, D.A., R.L Haedrich \& G.A. Rose. 2001. The fish assemblage of a Newfoundland estuary: diel monthly and annual variation. J. Fish Biol. 52: 669-687.

Miller, R.R., W.L Minckley \& S.M. Norris. 2005. Freshwater fishes of México. Universidad de Chicago, Chicago, Illinois, EEUU.

Mistch, W.J. \& J.G. Gosselink. 2000. Wetlands, p. 621-658. In R. Van Nostrands, R.J. Naimam \& H. Descamps (eds.). The ecology of interfaces: Riparian zones. An. Rev. Ecol. Syst., Nueva York, EEUU.

Moguel, O.E.J. \& M.J.F. Molina-Enríquez. 2000. La precipitación Pluvial en Tabasco y Chiapas. Rev. Kuxulkab. Univ. Juárez. Autón. Tabasco 510: 1-8.

MVSP. 1985-2000. Multivariate statistical package. Version $3.12 b$. Kovach Computing Services.

Norma Oficial Mexicana NOM-059-ECOL-2001. Protección ambiental-especies nativas de México de flora y fauna silvestres-categorías de riesgo y especificaciones par su inclusión, exclusión o cambio-lista de especies en riesgo. Diario Oficial de la Federación, 6 de Marzo de 2002, México.

Noiset, J.L. \& A.S. Hernández. 1991. Valorisation des marais par le développement de la pêche dans la région de San Pedro (Tabasco, Mexique). Ass. Dével. Rech. Agr. Int., Louvain-la-Neuve, Bélgica.

Páramo, D.S. 1984. Ictiofauna del río González y lagunas adyacentes, Tabasco. México. Univ. Cienc., Univ. Juárez. Autón. Tabasco 1: 5-19.

Peña-Mendoza, B., J.L. Gómez-Marquéz, I.H. SalgadoUgarte \& D. Ramírez-Noguera. 2005. Reproductive biology of Oreochromis niloticus (Perciformes: Cichlidae) at Emiliano Zapata dam, Morelos, México. Rev. Biol. Trop. 53: 515-522.

Pereyra-Díaz, D., U.B. Murrieta \& M.A.N. Baizabal. 2004. Influencia de la niña y el niño sobre la precipitación de la ciudad de Villahermosa Tabasco, México. Univ. Cienc., Univ. Juárez. Autón. Tabasco 20: 33-38.

Petry, P., P.B. Bayley \& D.F. Markle. 2003. Relationships between fish assemblages, macrophytes and environmental gradients in the Amazon River floodplain. J. Fish Biol. 63: 547-579.

Pielou, E.C. 1966. The measurement of diversity in different types of biological collections. J. Theor. Biol. 13: $131-350$.

Quiroz, C.H., G.J. Rodríguez, M.F.I. Astudillo, D.M. Vargas \& T.P. Jiménez. 2010. Condiciones abióticas de la presa El Abrevadero, utilizada para el cultivo extensivo de Oreochromis niloticus en Morelos, México. Rev. Electrón. Vet. 11: 1-19. (También disponible en línea: www.veterinaria.org/revistas/ redvet/n070710/071002.pdf).

Reséndez, M.A. \& B.M Salvadores. 2000. Peces de la Reserva de la Biosfera Pantanos de Centla. Resultados preliminares. Univ. Cienc., Univ. Juárez. Autón. Tabasco 15: 140-146. 
Rodiles-Hernández, R. \& A. González-Díaz. 2006. Ficha técnica de Thorichthys socolofi. In J.J. SchmitterSoto (ed.). Evaluación del riesgo de extinción de los cíclidos mexicanos y de los peces de la frontera sur incluidos en la NOM-059. El Colegio de la Frontera Sur (ECOSUR). Bases de datos SNIB-CONABIO. Proyecto No.CK001, México D.F., México.

Sáenz, I., M.Q. Protti \& J.P. Cabrera. 2006. Composición de especies y diversidad de peces en un cuerpo de agua temporal en el Refugio Nacional de Vida Silvestre Caño Negro, Costa Rica. Rev. Biol. Trop. 54: 639-645.

Schmitter-Soto, J.J. 1998. Catálogo de los peces continentales de Quintana Roo. Guías científicas ECOSUR. Colegio de la Frontera Sur, Chiapas, México.

Shannon, E.C. \& W. Wiener. 1963. The mathematical theory of communication. Universidad de Chicago, Illinois, EEUU.

Súarez, Y.R., M. Petrere Jr. \& A.C. Catella. 2004. Factors regulating diversity and abundance of fish communities in Pantanal lagoons, Brazil. Fish. Manag. Ecol. 11: $45-50$.

Sokal, R.R. \& F.J. Rohlf. 1981. Biometry. The principles and practice of statistics in biological research. W.H. Freeman and Co., San Francisco, California, EEUU.

StatSoft. 2004. STATISTICA. Data analysis software system. Version 7. Tulsa, Oklahoma, EEUU.

Stevenson, D.K. 1982. Una revisión de los recursos marinos de la región de la comisión de pesca para el atlántico centro occidental (COPACO). Doc. Téc. Pesca. FAO, Rome, Italy.
Taylor, C.M. \& M.L. Warren. 2001. Dynamics in species composition of stream fish assemblages: environment variability and nested subset. Ecology 82: 2320-2330.

Ter Braak, C.J.F. \& F.M. Verdonshot. 1995. Canonical correspondence analysis and related multivariate methods in Aquatic Ecology. Aquat. Sci. 57: 255-286.

Torres-Castro, I.L., M.E. Vega-Cendejas, J.J. SchmittterSoto, G. Palacio-Aponte \& R. Rodiles-Hernández. 2008. Ictiofauna de sistemas cársticos-palustres con impacto antrópico: los Peténes de Campeche, México. Rev. Biol. Trop. 57: 141-157.

Urriola, M., J. Cabrera Peña \& M.Q. Protti. 2004. Fecundidad fertilidad e índice gonadosomático de Poecilia reticulata (Pisces: Poeciliidae) en un estanque en Santo Domingo, Heredia, Costa Rica. Rev. Biol. Trop. 52: 945-950.

Valdez-Moreno, M.E., J.P. Pool-Canul \& S. ContrerasBalderas. 2005. A checklist of the Freshwater ichthyofauna from El Petén and Alta Verapaz, Guatemala, with notes for its conservation and management. Zootaxa 1072: 43-60.

Welcomme, R.L. 1985. River fisheries. FAO Fisheries Technical Paper: 262. Roma, Italia.

West, R.C., N.P. Psuty \& B.G. Thom. 1985. Las tierras bajas de Tabasco en el Sureste de México. Gobierno del Estado de Tabasco, México.

Winemiller, K.O. \& D.B. Jepsen. 1998. Effect of seasonality and fish movement on tropical river food webs. J. Fish. Biol. 53: 267-296.

Zar, J.H. 1999. Biostatistical analysis. Prentice-Hall, Nueva Jersey, EEUU. 